\title{
Translocação do Glyphosate em Biótipos de AZevém (Lolium multiflorum)
}

\author{
Glyphosate Translocation in Italian Ryegrass Biotypes (Lolium multiflorum)
}

FERREIRA, E.A. ${ }^{2}$, SANTOS, J.B. ${ }^{3}$, SILVA, A.A. ${ }^{4}$, OLIVEIRA, J.A. ${ }^{5}$ e VARGAS, L. ${ }^{6}$

\begin{abstract}
RESUMO - Foram avaliadas neste trabalho a absorção foliar e a translocação do glyphosate por biótipos de azevém (Lolium multiflorum) sensiveis e resistentes a esse herbicida. Para isso, aplicou-se ${ }^{14} \mathrm{C}$-glyphosate utilizando-se uma microsseringa de precisão, adicionando-se $10 \mu \mathrm{L}$ da calda sobre a face adaxial da primeira folha com lígula totalmente visível, quando as plantas de azevém se apresentavam com três perfilhos. A quantidade de glyphosate absorvido e translocado foi avaliada em intervalos de tempo $(2,4,8,16,32$ e 64 horas após a aplicação), por meio da medição da radiação emitida pelo ${ }^{14} \mathrm{C}$-glyphosate, em espectrômetro de cintilação líquida. Foram analisadas a parte aérea e as raízes, bem como a folha onde foi feita a aplicação e a solução de lavagem desta folha. A velocidade de absorção do glyphosate foi semelhante em ambos os biótipos de azevém, observando-se mais de 50\% de absorção desse herbicida nas primeiras oito horas após a aplicação. Maior retenção de glyphosate foi observada na folha tratada do biótipo resistente: $81,64 \%$ do total de glyphosate absorvido até as 64 horas. No biótipo sensivel esse valor foi de 55\% no mesmo período. No restante da parte aérea e nas raízes, a maior quantidade do glyphosate absorvido foi encontrada no biótipo sensível, mostrando sua maior capacidade de translocação. Após 64 horas da aplicação do glyphosate, apenas 6\%, em média, do glyphosate se encontrava nas plantas, indicando que a maior parte do produto pode ter sido exsudada. Conclui-se que a sensibilidade do azevém ao glyphosate pode ser atribuída à maior capacidade de translocação desse herbicida pelo biótipo sensível.
\end{abstract}

Palavras-chave: absorção, radioquímico, resistência, translocação.

ABSTRACT - This work evaluated foliar absorption and glyphosate translocation in Italian ryegrass (Lolium multiflorum) susceptible and resistant biotypes, ${ }^{14} \mathrm{C}$-glyphosate was applied by using a precision micro syringe, and adding $10 \mu \mathrm{L}$ of solution on the adaxial side of the first leaf with ligule totally visible, when Italian ryegrass plants showed 3 tillers. The quantity of glyphosate absorbed and translocated was evaluated through a liquid scintilation spectrometer by measuring radiation emitted by ${ }^{14} \mathrm{C}$-glyphosate at 2, 4, 8, 16, 32 and 64 hours after application. The roots and aerial part were analyzed as well as the leaf where application was performed and the solution used to wash the leaf. Glyphosate absorption speed was similar in both Italian ryegrass biotypes, with over 50\% of herbicide absorption in the first 8 hours after application. Up to 64 hours, higher retention (81.64\%)of glyphosate was observed in the resistant biotype, while only 55\% was retained in the susceptible biotype. In the remaining aerial part and roots, a higher amount of glyphosate was absorbed by the susceptible biotype, indicating higher capacity of its translocation. After 64 hours of application, only 6\%, of glyphosate was found in the plants, on average indicating that most of the product could be exuded. It could be concluded that Italian ryegrass sensitivity to glyphosate can be attributed to higher translocation capacity of this herbicide by susceptible biotypes.

Keywords: absorption, radiochemical, resistance, translocation.

Recebido para publicação em 2.3.2006 e na forma revisada em 5.5.2006.

2 Eng.-Agrônomo, doutorando em Fitotecnia da Universidade Federal de Viçosa - DFT/UFV, 36570-000 Viçosa-MG, <evanderal@yahoo.com.br>; ${ }^{3}$ D.S., Bolsista Pós-doutor em Fitotecnia - DFT/UFV; ${ }^{4}$ D.S., Professor do Departamento de Fitotecnia - DFT/UFV; ${ }^{5}$ D.S., Professor do Departamento de Biologia Geral - DBG/UFV. ${ }^{6}$ D.S., Pesquisador da Embrapa Trigo, Caixa Postal 451, 99001-970 Passo Fundo-RS.

Planta Daninha, Viçosa-MG, v. 24, n. 2, p. 365-370, 2006 


\section{INTRODUÇÃO}

O glyphosate vem sendo usado intensivamente na agricultura mundial há mais de 25 anos; apesar disso, oito espécies de plantas daninhas (Amaranthus palmeri, Ambrosia artemisiifolia, Conyza canadensis, Conyza banariensis, Lolium rigudum, Eleusine indica, Lolium multiflorum, e Plantago lanceolata) adquiriram resistência a esse herbicida (Weed Science, 2006).

O azevém (Lolium multiflorum) é uma gramínea de ciclo anual, infestante das principais lavouras localizadas na região Sul do Brasil. O primeiro caso de resistência de $L$. multiflorum ao glyphosate foi confirmado no Chile por Perez \& Kogan (2002), os quais atribuíram o aparecimento de resistência ao uso repetido de glyphosate na área durante os últimos oito a dez anos.

De modo geral, o aparecimento de resistência a herbicidas é atribuído à absorção, translocação, compartimentalização e metabolização diferencial do herbicida pelas plantas. No caso específico de $L$. multiflorum, especula-se que a resistência adquirida possa ser devido à absorção e translocação diferencial do herbicida nas plantas resistentes.

A absorção de herbicidas é um processo bifásico que envolve rápida penetração inicial através da cutícula, seguida por absorção simplástica, sendo a duração desse processo dependente de vários fatores, como espécie e idade da planta, condições ambientais e concentração do herbicida e do surfatante (Silva et al., 2005).

Uma vez absorvido, é necessária a translocação do herbicida, através de tecidos vasculares, para os sítios-alvo, onde o produto vai exercer sua atividade herbicida (Satichivi et al., 2000). A translocação representa um processo essencial para a eficácia do herbicida (Wanamarta \& Penner, 1989).

O glyphosate se movimenta no floema seguindo a rota dos produtos da fotossintese, das folhas fotossinteticamente ativas (fonte) em direção às partes das plantas em crescimento (dreno), para manutenção do metabolismo e/ou formação de produto de reserva, das raízes, dos tubérculos, das folhas jovens e de zonas meristemáticas (Hetherington et al., 1998).
Dessa forma, a absorção, a translocação e o metabolismo do herbicida podem afetar a sensibilidade de uma planta, pois este precisa atingir o sítio de ação numa concentração adequada.

Objetivou-se neste trabalho teve como objetivo avaliar a absorção e a translocação do glyphosate por plantas de azevém sensiveis e resistentes a esse herbicida, visando desenvolver técnicas para o manejo dessa espécie em lavouras onde o principal produto eficiente para o seu controle era o glyphosate.

\section{MATERIAL E MÉTODOS}

Para realização deste trabalho cultivaram-se plantas de azevém (biótipos resistente e sensivel em vasos contendo $200 \mathrm{~mL}$ de substrato composto de solo e areia lavada na proporção de 3:1 e adubado) em câmara de crescimento com temperatura dia/noite de $25 / 16{ }^{\circ} \mathrm{C}$, respectivamente, e fotoperíodo de 12 horas de luz.

$\mathrm{O}{ }^{14} \mathrm{C}$-glyphosate foi aplicado em mistura com glyphosate comercial (800 g ha-1 do e.a.) sobre a face adaxial da primeira folha com lígula totalmente visível, utilizando-se uma microsseringa, adicionando-se $10 \mu \mathrm{L}$ da calda com atividade específica de aproximadamente $700 \mathrm{~Bq}$, quando as plantas apresentavam três perfilhos ou 30 dias após a emergência.

A avaliação da absorção e translocação do ${ }^{14} \mathrm{C}$-glyphosate em biótipos resistente e sensivel foi feita em diferentes intervalos de tempo (2, $4,8,16,32$ e 64 horas após o tratamento com o produto). Após cada período de exposição, as plantas foram coletadas e fracionadas em folha de aplicação, parte aérea e raízes. A folha de aplicação foi lavada com 9,0 mL de metanol, para extração do produto não absorvido. Todo o material vegetal foi seco em estufa a $50{ }^{\circ} \mathrm{C}$, por 48 horas, para obtenção da massa seca de cada componente. Em seguida, procedeu-se à moagem de cada componente em moinho de bola, até uma textura equivalente a 200 mesh. Depois disso, amostras de $100 \mathrm{mg}$ de massa seca de cada componente da planta (folha de aplicação, parte aérea e raízes) foram adicionadas em frascos de $20 \mathrm{~mL}$, para contagem. Elas foram homogeneizadas com $1 \mathrm{~mL}$ de Triton-X-100 por 14 segundos em vortex, adicionando, em seguida, $5 \mathrm{~mL}$ de coquetel de cintilação, sendo novamente submetidas à 
homogeneização. Os frascos assim preparados foram colocados em espectrômetro de cintilação líquida Beckman 6500, o qual corrigia o quenching automaticamente.

Os valores da radiação (cpm total) encontrada na solução de lavagem e na planta foram convertidos em porcentagem em relação à radiação total aplicada. Na planta, esses valores de ${ }^{14} \mathrm{C}$-glyphosate foram convertidos em porcentagem em relação ao total presente na planta e distribuídos na folha tratada, parte aérea e raízes. Os resultados foram apresentados em gráficos, utilizando-se médias observadas e desvios-padrão.

\section{RESULTADOS E DISCUSSÃO}

A avaliação do ${ }^{14} \mathrm{C}$-glyphosate residual da solução de lavagem nas folhas tratadas dos biótipos de azevém resistente e sensivel mostrou valores semelhantes, exceto às 4 HAT (horas após aplicação dos tratamentos), quando a absorção do ${ }^{14} \mathrm{C}$ glyphosate foi maior no biótipo sensivel. Nos demais intervalos avaliados $(2,8,16,32$ e 64 HAT) foi observada maior absorção do produto pelo biótipo sensivel, porém sem diferença significativa (Figura 1), indicando possivel variação na taxa de absorção do glyphosate pelos diferentes biótipos. Resultados semelhantes também foram observados por Lorraine-Colwill et al. (2002), que não constataram diferenças na capacidade de absorção de glyphosate pelos biótipos resistente e sensível de azevém. Nas primeiras 8 HAT observou-se rápida absorção em ambos os biótipos (Figura 1), evidenciada pela redução do ${ }^{14} \mathrm{C}$-glyphosate avaliada na solução de lavagem, o que correspondeu a 48,79 e 61,09\% de absorção nos biótipos resistente e sensivel, respectivamente. Das 8 às 64 HAT a velocidade de absorção foi mais lenta, atingindo 75,02 e 76,64\% nos biótipos resistente e sensivel, respectivamente, ao final de 64 HAT (Figura 1). Estudos realizados em diferentes culturas e condições experimentais têm mostrado porcentagens de absorção foliar do glyphosate distintas, variando de 6 a $70 \%$ (Bariuan et al., 1999; Hetherington et al., 1999; Hall et al., 2000; Kirkwood et al., 2000; Reddy, 2000; Satchivi et al., 2000; Sharma \& Singh, 2001; Pline et al., 2001). Em Sorghum halepenses e Elytrigia repens a absorção variou de 7 a $74 \%$ durante os períodos de 48 e
120 horas de exposição (Mcwhorter et al., 1980; Devine et al., 1983). Resultado semelhante foi observado por Monquero et al. (2004), os quais constataram absorção de 66,80 e 90\% do glyphosate aplicado nas espécies Commelina benghalensis, Ipomoea grandifolia e Amaranthus hybridus, respectivamente, após 72 horas da aplicação dos tratamentos.

Com relação à concentração de ${ }^{14} \mathrm{C}$ glyphosate na folha de aplicação, observou-se maior acúmulo de ${ }^{14} \mathrm{C}$-glyphosate no biótipo resistente em relação ao sensivel. Verificou-se que, no biótipo resistente, decorridas 64 horas da aplicação do produto, aproximadamente $81,64 \%$ do total absorvido encontrava-se retido na folha onde foi feita a aplicação, enquanto no biótipo sensivel a retenção foi de 54,66\% (Figura 2). Monquero et al. (2004), verificaram que valores próximos de

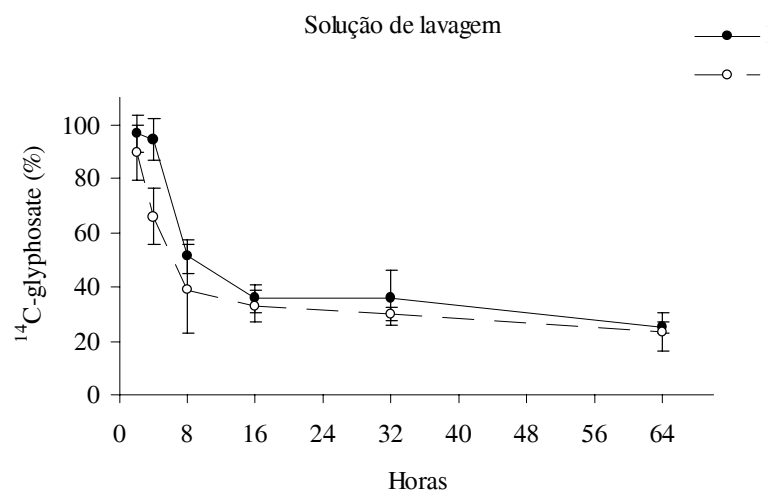

Figura 1 - Porcentagem de ${ }^{14} \mathrm{C}$-glyphosate na solução de lavagem, em relação ao total aplicado, nos biótipos resistente (R) e sensível (S).

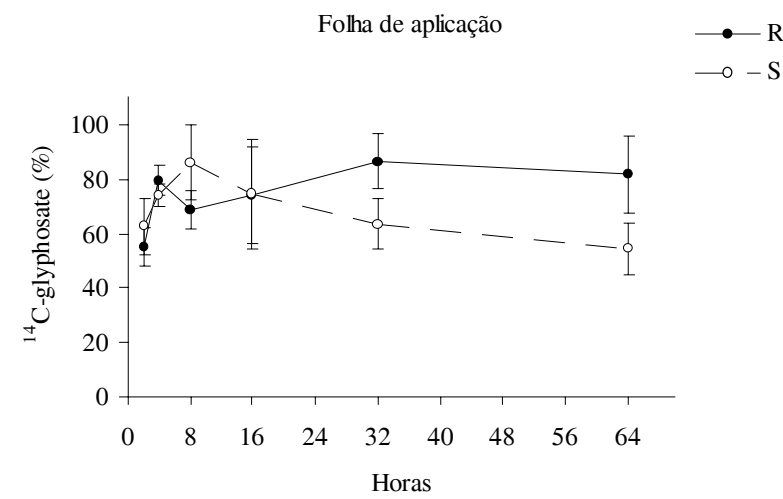

Figura 2 - Porcentagem de ${ }^{14} \mathrm{C}$-glyphosate na folha de aplicação, em relação ao total presente na planta, nos biótipos resistente (R) e sensível (S).

Planta Daninha, Viçosa-MG, v. 24, n. 2, p. 365-370, 2006 
$68 \%$ do ${ }^{14} \mathrm{C}$-glyphosate aplicado se encontravam na folha tratada às 72 HAT nas plantas de Commelina benghalensis, Ipomoea grandifolia e Amaranthus hybridus. Maior retenção de glyphosate na folha de biótipo resistente de Lolium rigidum também foi relatada por Lorraine-Colwill et al. (2002), os quais observaram maior acúmulo de ${ }^{14} \mathrm{C}$ glyphosate nas pontas das folhas tratadas do biótipo resistente de L. rigidum, em relação ao biótipo sensivel. De modo geral, ocorreu grande retenção do glyphosate nas folhas que receberam o tratamento; provavelmente, esse fato se deve à ação do produto, que interrompe o ciclo do carbono no cloroplasto, causando redução na síntese de carboidratos, diminuindo o transporte destes para os drenos e, conseqüentemente, diminuindo o transporte de glyphosate (McAllister \& Haderlie, 1985; Satchivi et al., 2000).

Duas horas após a aplicação do ${ }^{14} \mathrm{C}$ glyphosate observou-se que esse produto já havia se distribuído pela parte aérea tanto no biótipo resistente $(22,35 \%)$ quanto no sensivel $(18,67 \%)$ (Figura 3). Todavia, às 64 HAT observou-se que esse valor foi reduzido para $10,33 \%$ no biótipo resistente, ao passo que no sensível ele foi de $18,05 \%$. O biótipo de azevém sensivel apresentou maior concentração do ${ }^{14} \mathrm{C}$-glyphosate na parte aérea entre 32 e 64 HAT (Figura 3). O movimento do glyphosate está associado aos carboidratos que governam a distribuição do herbicida na planta, pois ocorre maior acúmulo desse herbicida nos ápices e em raízes (McAllister \& Haderlie, 1985).

Nas raízes foi encontrada distribuição de glyphosate semelhante à observada na parte aérea, onde, duas horas após a aplicação, 22,75 e $18,82 \%$ de todo o ${ }^{14} \mathrm{C}$-glyphosate presente nas plantas foram detectados nas raízes dos biótipos resistente e sensivel, respectivamente (Figura 4). Os resultados, portanto, indicam que esse herbicida se movimenta muito rápido pela planta, e esse movimento está associado às velocidades de transporte de açúcares no floema, que são elevadas e, em geral, podem variar de 0,3 a $1,5 \mathrm{~m} \mathrm{~h}^{-1}$ (Taiz \& Zeiger, 2004). Fazendo comparação entre os biótipos, às 64 horas após a aplicação, verificou-se maior concentração do herbicida no biótipo sensível na parte aérea e nas raízes, enquanto no resistente a maior quantidade do produto se encontrava na folha aplicada, indicando menor translocação do ${ }^{14} \mathrm{C}$-glyphosate no biótipo resistente (Figura 4). Resultados semelhantes foram observados por LorraineColwill et al. (2002), que verificaram, após 48 horas da aplicação do ${ }^{14} \mathrm{C}$-glyphosate, acúmulo de 20 e $6 \%$ nas raízes de biótipos sensivel e resistente, respectivamente, em plantas de Lolium rigidum.

Durante todos os intervalos avaliados, observou-se que o percentual total de herbicida marcado nos dois biótipos variou de 3 a 6\% (Figura 5). Também foi observado que a concentração do ${ }^{14} \mathrm{C}$-glyphosate da solução de lavagem decaiu de aproximadamente $90 \%$ nos dois biótipos para valores próximos a 30\% (Figura 1), e o total medido (na solução de aplicação e na planta) apresentou o mesmo comportamento, reduzindo para aproximadamente $30 \%$ em ambos os biótipos (Figura 6). Independentemente da distribuição do glyphosate pelos biótipos de azevém, verificouse que às 64 HAT grande parte do produto aplicado (70\%, em média) não se encontrava mais presente no material (Figura 6), indicando sua possível eliminação pelas plantas. Também, Monquero et al. (2004) constataram que, do total do ${ }^{14} \mathrm{C}$-glyphosate aplicado nas espécies Commelina benghalensis, Ipomoea grandifolia e Amaranthus hybridus, em 72 HAT apenas $2,2,3,5$ e 4,6\% do produto se encontravam nas plantas. Provavelmente parte da redução do ${ }^{14} \mathrm{C}$-glyphosate na planta com o decorrer do tempo deve-se à exsudação radicular. Diversos autores têm observado a exsudação do glyphosate em cultivos celulares (Hetherington et al., 1998), e, em experimentos onde o glyphosate é aplicado nas folhas, seus efeitos são observados nas plantas adjacentes, que compartilham a mesma zona radicular (Coupland \& Lutman, 1982). Linder et al. (1964) observaram que algumas plantas são capazes de exsudar no solo, em pouco tempo, substâncias aplicadas via foliar. Rodrigues et al. (1982) comprovaram a exsudação radicular de ${ }^{14} \mathrm{C}$-glyphosate por plantas de trigo tratadas com esse herbicida e que plantas de milho cultivadas próximo ao trigo absorviam o ${ }^{14} \mathrm{C}$ - glyphosate pelas raízes, distribuindo-o por todas as partes da planta, sendo as maiores concentrações encontradas nas raízes. 
Na Figura 7 verifica-se que, cinco dias após a aplicação do glyphosate, as plantas sensíveis se encontravam completamente secas. Considerando que esse herbicida foi aplicado em uma única folha, é possível comprovar

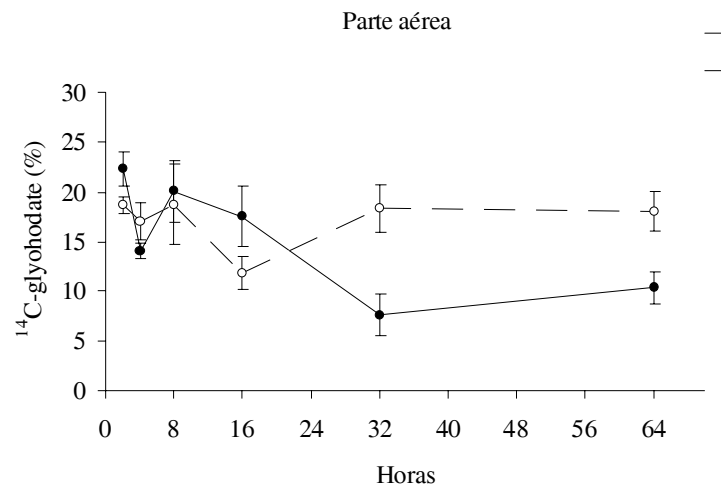

Figura 3 - Porcentagem de ${ }^{14} \mathrm{C}$-glyphosate na parte aérea, em relação ao total presente na planta, nos biótipos resistente (R) e sensível (S).

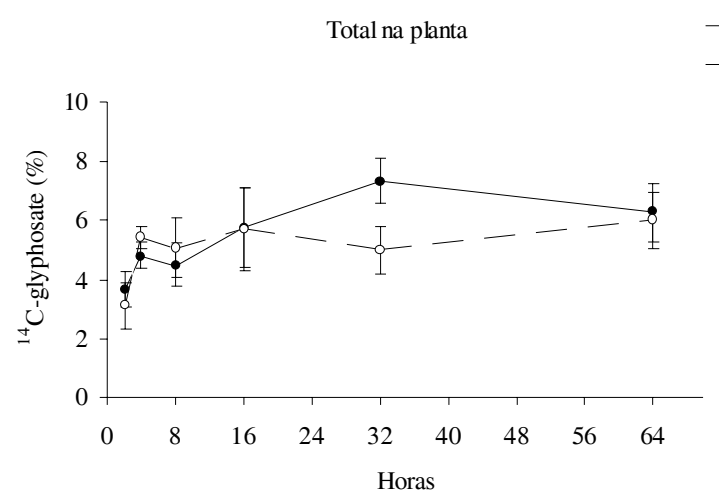

Figura 5 - Porcentagem de ${ }^{14} \mathrm{C}$-glyphosate na planta, em relação ao total aplicado, nos biótipos resistente (R) e sensível (S). a rápida translocação do produto por toda a planta, observando-se morte de todos os perfilhos, ao passo que o biótipo resistente não apresentou sintomas aparentemente visiveis.

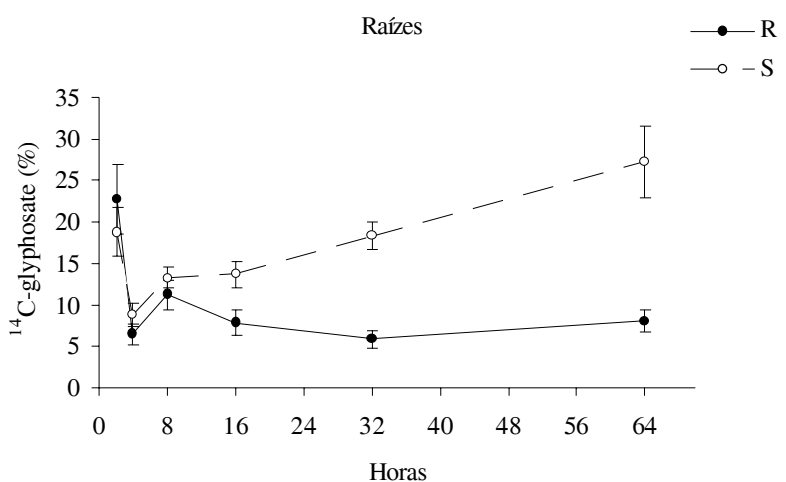

Figura 4 - Porcentagem de ${ }^{14} \mathrm{C}$-glyphosate nas raízes, em relação ao total presente na planta, nos biótipos resistente $(\mathrm{R})$ e sensível (S).

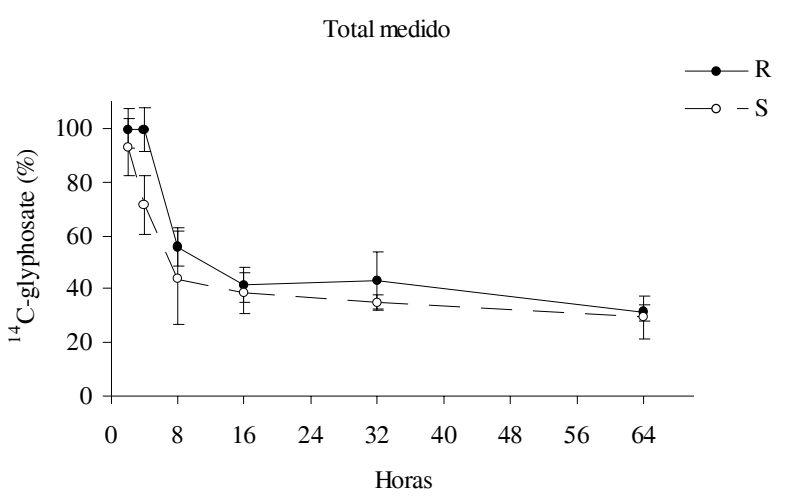

Figura 6 - Porcentagem de ${ }^{14} \mathrm{C}$-glyphosate medido (solução de lavagem + planta), em relação ao total aplicado, nos biótipos resistente (R) e sensível (S).

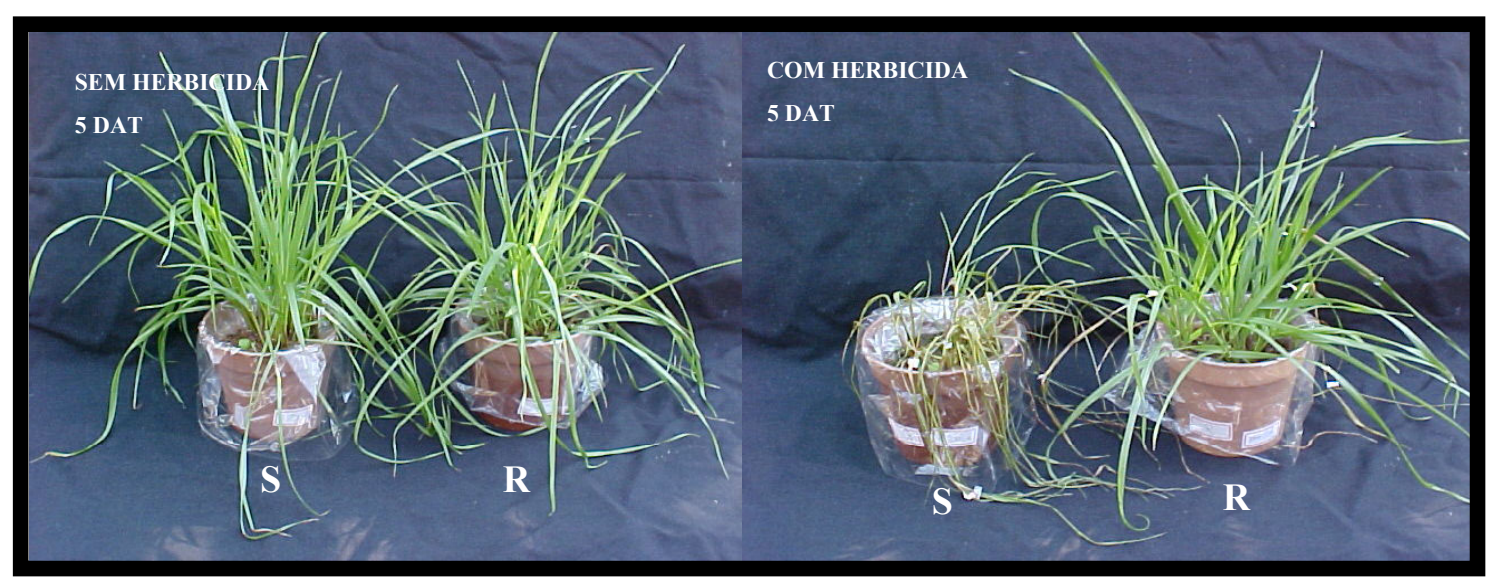

Figura 7 - Biótipos sensível e resistente, sem e com herbicida, aos cinco dias após a aplicação dos tratamentos. 
Os resultados obtidos no presente trabalho permitem concluir que os biótipos apresentam diferenças marcantes com relação à translocação do ${ }^{14} \mathrm{C}$-glyphosate. O mecanismo de resistência de $L$. multiflorum pode estar ligado à translocação diferencial desse herbicida nos biótipos. Os resultados mostram menor taxa de distribuição do produto no biótipo resistente, no qual se observou maior acúmulo do produto marcado na folha tratada. No biótipo sensível foi constatado maior acúmulo na parte aérea e nas raízes. Foi observada pequena diferença na absorção quatro horas após a aplicação dos tratamentos, com o biótipo sensível absorvendo maior quantidade do produto; contudo, nas primeiras duas horas e após as oito horas não houve diferença entre os tratamentos.

\section{AGRADECIMENTOS}

Ao Conselho Nacional de Desenvolvimento Científico e tecnológico (CNPq), pelo apoio financeiro, e à MONSANTO, pelo fornecimento do ${ }^{14} \mathrm{C}$-glyphosate.

\section{LITERATURA CITADA}

BARIUAN, J.; REDDY, K.; WILLS, G. Glyphosate injury, rainfastness, absorption, and translocation in purple nutsedge (Cyperus rotundus). Weed Technol., v. 13, p. 112-119, 1999.

COUPLAND, D.; LUTMAN, P. Investigations into the movement of glyphosate from treated to adjacent untreated plants. Ann. Appl. Biol., v. 101, p. 315-321, 1982.

DEVINE, M. D.; BANDEEN, J. D.; MCKERSIE, B. D. Temperature effects on glyphosate absorption, translocation and distribution in quackgrass (Agropyron repens). Weed Sci., v. 31, p. 461-464, 1983.

HALL, G.; HART, C.; JONE, C. Plants as source of cations antagonistic to glyphosate activity. Pest Manag. Sci., v. 56, p. $351-358,2000$.

HETHERINGTON, P. et al. The absorption, translocation and distribution of the herbicide glyphosate in maize expressing the CP-4 transgene. J. Exp. Bot., v. 50, p. $1567-1576,1998$.

KIRKWOOD, R. et al. Absorption, localization, translocation and activity of glyphosate in barnyardgrass (Echinochloa crus-galli (L) Beauv): influence of herbicide and surfactant concentration. Pest Manag. Sci., v. 56, p. $359-367,2000$.
LINDER, P. J.; MITCHELL, J. W.; FREEMAN, G. D. Persistence and translocation of exogenous regulating compounds that exude from roots. J. Agric. Food Chem., v. 12 , p. $437-438,1964$.

LORRAINE-COLWILL, D. F. et al. Investigations into the mechanism of glyphosate resistance in Lolium rigidum. Pest. Biochem. Physiol., v. 74, p. 62-72, 2002.

MCWHORTER, C.; JORDAN, T.; WILLS, C. Translocation of ${ }^{14} \mathrm{C}$-glyphosate in soybeans (Glycine max) and johnsongrass (Sorghum halepense). Weed Sci., v. 28, p. 113-118, 1980.

MCALLISTER, R.; HABERLIE, L. L. Translocation of ${ }^{14} \mathrm{C}$-Glyphosate and ${ }^{14} \mathrm{CO}$ - Labeled photoasimilates in canada thistle (Cirsium arvense). Weed Sci., v. 33, p. 153-159, 1985.

MONQUERO, P. A. et al. Absorção, translocação e metabolismo do glyphosate por plantas tolerantes e suscetíveis a este herbicida. Planta Daninha, v. 22, p. 445-451, 2004.

PEREZ, A.; KOGAN, M. Glyphosate-resistant Lolium multiflorum in Chilean orchards. Weed Res., v. 43, p. 12-19, 2002.

PLINE, W. et al. Absorption and translocation of glyphosate in glyphosate-resistant cotton as influenced by application method and growth stage. Weed Sci., v. 49, p. 460-467, 2001.

REDDY, K. Factors affecting toxicity, absorption, and translocation of glyphosate in redvine (Brunnichia ovata). Weed Technol., v. 14, p. 457-462, 2000.

RODRIGUES, J. J. V.; WORSHAM, A. D.; CORBIN, F. T. Exudation of glyphosate from wheat (Triticum aestivum) plants and its effects on interplanted corn (Zea mays) and soybeans (Glycine max). Weed Sci., v. 30, p. 316-320, 1982.

SATICHIVI, N. et al. Absorption and translocation of glyphosate isopropylamine and trimethysulfonium salts in Abutilon theophrasti and Setaria faberi. Weed Sci., v. 48, p. 675-679, 2000.

SHARMA, S.; SINGH, M. Environmental factors affecting absorption and bio-efficacy of glyphosate in florida beggarweed (Desmodium tortuosum). Crop Protec., v. 20, p. 511-516, 2001.

SILVA, A. A.; FERREIRA, F. A.; FERREIRA, L. R. Controle de plantas daninhas. Brasília: ABEAS, 2005. 260 p.

WANAMARTA, G. D.; PENNER, D. Foliar absorption of herbicides. Weed Sci., v. 4, p. 215-231, 1989.

TAIZ, L.; ZEIGER, E. Fisiologia vegetal. 3.ed. São Paulo: Artmed, 2002. 719 p.

WEED SCIENCE - INTERNATIONAL SURVEY OF HERBICIDE RESISTANT WEEDS. Disponível em: <http:// www.weedscience.org/in.asp>. Acesso em: 17 jan. 2006. 